\title{
Physical Activity for Individuals with Cerebral Palsy
}

\author{
Jonathon Law a, Colin G. Pennington ${ }^{a,{ }^{*}}$
}

a Department of Sports ScienceSchool of Kinesiology -- College of Education, Tarleton State University, TX, 76036

*Corresponding Author Email: cpennington@tarleton.edu

DOI: https://doi.org/10.34256/ijpefs2129

Received: 23-03-2021, Revised: 15-06-2021; Accepted: 16-06-2021; Published: 21-06-2021

Abstract: Despite the fact that cerebral palsy affects the balance, coordination, muscles, and movements of individuals, adaptive sports can be used to help individuals with cerebral palsy stay physically active. High-quality physical education and movement opportunities can integrate children with cerebral palsy into movement programing, as to enable children with cerebral palsy to achieve goals related to cognitive skills associated with exploring physical health, maintaining physical health, as well as affective values towards physical health, movement, play, and general wellbeing. Cerebral palsy has not prevented some people from being athletes who have managed to compete on the international stage. Sports is one way to encourage people with cerebral palsy to socialize, be fit, stay healthy, and, most importantly, have fun. Enjoyment of physical activity can improve the selfefficacy of individuals with cerebral Palsy. It can motivate them to override pain and sustain their participation. This article details the considerations, recommendations, safety concerns, and facilitation protocols for individuals with cerebral palsy participating in adapted sport.

Keywords: Cerebral palsy, Adaptive sport, Special Olympics, Paralympics, Inclusive physical activity

Jonathan Law is a graduate student of Kinesiology at Tarleton State University. Law will be entering the health and movement profession upon completion of his degree where he will work with students, athletes, and the general public.

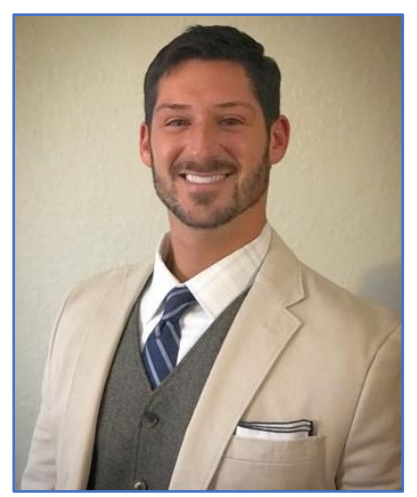

Colin G. Pennington (PhD) is an Assistant Professor of Kinesiology at Tarleton State University where he works with Exercise and Sport Study majors, and carries out research on physical education teacher effectiveness and other pedogogical and healthrelated applications of the kinesiology sub-disciplines. His interests and research focus on teacher socialization, physical education teacher training, character development programs within physical education and sport, and health and wellness.

\section{Introduction}

Cerebral palsy is the most common motor disability in childhood and can significantly impact the amount of physical activity and individual obtains. Furthermore, the scope and quality of the physical activity may be drastically limited by cerebral palsy. High-quality physical education and movement opportunities can integrate children with cerebral palsy into movement programing, as to enable children with cerebral palsy to achieve goals related to cognitive skills associated with exploring physical health, maintaining physical health, as well as affective values towards physical health, movement, play, and general wellbeing [1]. Despite the well-researched and wellestablished physical activity recommendations for adults and children, detailed recommendations for physical activity and sedentary behavior have not been established for children, adolescents, and adults with cerebral palsy [2]. One may correctly assume that children and young people with cerebral palsy are far less active than those without cerebral palsy. Frankly, this is because children with cerebral palsy face a greater number of, and different, barriers to accessing and participating in physical activity than "typically developing" children [3]. For example, a family with a 
child with cerebral palsy "may experience diverse contextual barriers such as the parent's fear of their child being injured or perceived negative attitudes in the community" [3].

Using data, theory, and philosophy from the worlds of medicine [4], adaptive adolescent psychology $[5,6]$, and adaptive sport pedagogy $[7,8]$, this article seeks to correct the imbalance of physical activity adoption for children with cerebral palsy by providing movement practitioners with concrete strategies and suggestions for modifying physical activity to be inclusive.

\section{Cerebral Palsy and Adaptive Sports}

Even though cerebral palsy affects the balance, coordination, muscles, and movements of individuals, adaptive sports can be used to enable those with cerebral palsy stay physically active [9]. Cerebral palsy has not prevented some people from being athletes who have managed to compete on the international stage. Sports is one way to encourage people with cerebral palsy to socialize, be fit, stay healthy, and, most importantly, have fun. Enjoyment of physical activity can improve the self-efficacy of individuals with cerebral Palsy. It can motivate them to override pain and sustain their participation.

Sport cannot fulfill the needs of everyone, which applies even to those with no disability. However, it is essential to make sports adaptive for people living with cerebral palsy to participate. For instance, an individual with cerebral palsy may be unable to run down and shoot baskets on a basketball court. However, if they are trained to use the mobility of a wheelchair, they can still dribble and shoot baskets [10]. This can only be achieved if society provides opportunities for people living with cerebral palsy to participate in sports. If communities create wheelchair basketball teams, then it will encourage participation. It is also crucial to understand that there are other adaptations that are even simpler to implement. For instance, a girl with cerebral palsy can be given two lanes to swim because of her movement difficulties. This can encourage her to compete with non-disabled children. This is the essence of adaptive sports.

\section{Special Olympics}

There are various ways adaptive sports can be implemented, from using special technologies for snowboarding or wheelchair racing to playing ice hockey [11]. Living with cerebral palsy does not mean that an individual is intellectually disabled. The opportunity to participate in Olympics can improve selfconfidence and encourage positive growth that comes from the competition. Participants can take advantage of such opportunities to develop their leadership skills and use the Olympics as a chance to mentor their counterparts living with cerebral palsy. Special Olympics was created in order to change attitudes about the intellectual capabilities of people living with disabilities. It has always aimed at promoting fitness and health for everyone with the primary intention of building strong communities.

\section{Paralympics}

The Paralympics is an international organization formed to mirror the Olympics, but only for participants living with physical disabilities [12]. Athletes are allowed to participate in competitions, such as wheelchair tennis, swimming, rowing, badminton, wheelchair racing, and many others. Paralympics gives people with cerebral palsy the opportunity to participate. The competition also encourages them to dream like other children around the world. An example of a person that should serve as a role model for people with cerebral palsy is Sophia Warner [13]. She started training to be a Paralympic sprinter at the age of 19 . She became a world champion despite living with cerebral palsy. Her engagement in sports and physical fitness motivated her to be a champion.

\section{Fact Sheet}

Physical fitness promotes a state of health where an individual can complete daily tasks with less exhaustion. Why should people with cerebral palsy engage in physical fitness?

1. Muscle fitness - It is the ability of the body to sustain activities that involve lifting heavy objects [14]. The muscles develop endurance by exerting force for an extended period. Muscle fitness requires consistency and a good diet.

2. Cardiorespiratory Fitness - It is the ability of the lungs and heart to circulate oxygen in the body of an individual [15]. This type of fitness is beneficial in increasing the level of endurance by engaging in physical activities for an extended period.

3. Joint Range of Motion or Flexibility - It prevents injury and facilitates the body's efficient movement at any single or group of joints [16]. 


\section{So Why Exercise?}

Research shows that a combination of joint range motion, cardiorespiratory, and muscular fitness can assist people with cerebral palsy achieve the following:

- Lower the risk of heart attack, stroke, cardiovascular illnesses, and hypertension.

- Reduce the risk of back pain.

- Improve stamina and reduce the risk of falling.

- Reduce the risk of type 2 diabetes.

- Reduce the risk of having weak bones that can lead to fracture.

- Encourage them to maintain a healthy weight.

- Improve the quality of life by reducing the risks of depression, stress, and low self-esteem [9].

- Improve functional mobility and capacity.

\section{Things to Consider Before Starting to Exercise}

Recommendations. The American College of Sports Medicine provides the following recommendations for people living with cerebral palsy [17].

1. For cardiorespiratory fitness activity, individuals should dedicate 150 minutes of weekly physical exercises of moderate intensity.

2. Muscle fitness should be performed at least two days per week for beginners.

However, it is worth noting that being fit should not be about all or nothing. This is because physical activity might be demanding, depending on the severity of cerebral palsy. This why achieving less than the recommendations can still lead to positive outcomes. The goal should be to start small to gain familiarity with both resistance and aerobic exercises.

\section{How to Get Started}

1. Consulting a physician - Individuals with cerebral palsy must consult with a physician before engaging in exercise activity. This is because the condition has different levels of severity [18]. This will enable trainers to modify activities to meet the physical abilities and individual needs of people with cerebral palsy. A healthcare professional must also review how medications might respond to particular body exercises.

2. Choose the preferred environment - Physical activity should be performed comfortably. Therefore, an individual's preference for the training environment is key to achieving positive outcomes. Some might want to train at home alone, while others prefer being in a group.
3. Setting a realistic goal - It is essential to select a particular time to perform routine exercises. Individuals should also be consistent in engaging in physical activities to achieve a high success rate.

4. Starting slowly - Physical activity requires patience, which is why there should be no hurry to achieve immediate results. Starting slowly gives the body the momentum of increasing the intensity of exercise. It is also a method of avoiding straining the body and preventing injury.

\section{Teaching Tips for Endurance and Muscle Strength, Cardiorespiratory, and Joint Range of Motion Exercises}

\section{Endurance and Muscle Strength}

1. Muscle rest - Straining the body's muscles can lead to injury. Exercise programs should provide an adequate resting period for muscles to rest [14]. This is why every exercise session must focus on different muscle groups, such as legs, arms, and shoulders. Regardless of the preference of the trainee, rest is an integral part of physical activities.

2. Focus on resistance, not on repetitions - It is important to understand that muscle strength is achieved by more weight resistance. Therefore, having many repetitions with the same weight makes an exercise too easy. For instance, one can start with a specific weight for 10 repetitions. After some time, add more weight for the same number of repetitions in order to improve endurance levels.

\section{Cardiorespiratory Exercise}

1. Heart rate- The primary goal of this fitness activity is to improve heart rate levels. The target zone should be between $40 \%$ and $85 \%$ of an individual's maximum heart rate.

2. Calculating the maximum heart rate - The maximum heart rate's target zone should be calculated in relation to age. Therefore, if an individual is 30 years old, the maximum heart rate would be $220-30=190$. This means that if the target zone for maximum heart rate is 40 to $85 \%$, then the range would be $76-162$ beats/minute. Therefore, it is recommended in this case to start at 72 beats/minute while progressing slowly until the maximum rate of 162 beats/minute is achieved.

3. Checking pulse rate - In order to ensure an individual is exercising in the target heart zone, record the pulse rate for 10 seconds, then multiply 
the results by 6 . If the heart rate is too low, it indicates that an individual should work harder.

4. Duration - 10 minutes is the recommended maximum duration for cardiorespiratory exercises.

\section{Joint Range of Motion}

1. Stretching and flexibility exercises can be performed before and during muscle fitness. This can be in the form of a warm-up before muscle or aerobic training. After finishing exercising, joints and muscles are usually warm. Therefore, it is advised to conclude with an effective stretching routine.

2. Dynamic Stretches - Joint range motion is influenced by muscle strength and length. A full range of motion can strengthen even muscles that are prone to injury during physical exercises. The goal should be to prevent injury and improve the function of joints.

\section{Health and Safety Precautions}

1. It is crucial for people with cerebral palsy to monitor how their bodies respond to exercises. Some danger signals that should be noted include severe joint and muscle pains, nausea, sudden weakness, excessive fatigue, breathing difficulty, chest pains, and light-headedness.

2. It is normal for first-time learners to experience mild soreness of muscles for up to 48 hours. Soreness is usually expected after exercises such as squatting down, walking downstairs, and lowering weight because they stretch the muscles.

3. Protective and comfortable clothing is always recommended. Wear a helmet before going for bicycle riding. It is also vital to wear attire that does not restrict physical activity.

\section{Adaptive Exercise Activities}

1. For cardiorespiratory fitness, it is recommended to ride a stationary bicycle. Suppose an individual is experiencing difficulty placing their feet on the pedals. In that case, a rubber strap or ace wrap can be used. A tricycle would be a better option for those struggling to ride a bicycle.

2. Upper extremity ergometers can be used to support people having difficulty in using their legs. For instance, the handles of bicycles can be positioned on the upper body for arm use.

3. Exercise bands with the recommended resistances or elastic capacities should be used to strengthen muscles while sitting, lying down, or standing.
4. While climbing stairs, a handrail can be used for support and safety.

5. The buoyancy and excellent resistance of water are beneficial to the body. Therefore, regular exercises can be performed in water.

6. Subscribe to adaptive exercise programs in community colleges, such as fitness classes, dance, yoga, and adaptive sports.

\section{The Role of Technology}

Technology has been instrumental in the development of adaptive equipment for people living with cerebral palsy. The adaptive devices are intended to improve the ability of individuals to function independently. It is worth noting that cerebral palsy can cause a different range of disabilities related to mobility, communication, and hearing. For individuals with mobility difficulties, low-tech non-electric wheelchairs and walkers can be used. Electric wheelchairs can assist individuals who struggle to move their arms (18Baldi et al., 2017). Lifts can also be used to facilitate their movement to different floors. For children with communication challenges, devices such as tablets and computers with special applications can be used. For instance, speech-generating applications can be integrated to make communication clearer. Cochlear implants can be used to assist children with hearing difficulties [19].

\section{Conclusion}

Cerebral palsy can limit individuals from performing more tasks than their non-disabled counterparts. However, they can also make a positive change in the world by engaging in sporting and physical activities. However, some guidelines should be followed to ensure that physical exercise improves their muscle strength, cardiorespiratory health, and mobility function. Sophia Warner is a great example that can motivate people living with cerebral palsy to believe that their abilities are limitless.

\section{References}

[1] C. Nelson, K. Fuchs, L.W. Pennington, \& C.G. Pennington, Cerebral Palsy: Enhancing Movement Opportunity with Help from the Care Team, International Journal of Physical Education, Fitness and Sports, 9(4) (2020) 2730. [DOI]

[2] O. Verschuren, M.D. Peterson, A.C. Balemans, \& E.A. Hurvitz, Exercise and physical activity 
recommendations for people with cerebral palsy, Developmental Medicine \& Child Neurology, 58(8) (2016) 798-808. [DOI] [PubMed]

[3] S. Reedman, R.N. Boyd, \& L. Sakzewski, The efficacy of interventions to increase physical activity participation of children with cerebral palsy: A systematic review and metaanalysis, Developmental Medicine \& Child Neurology, 59(10) (2017) 1011-1018. [DOI] [PubMed]

[4] P.G. McPhee, O. Verschuren, M.D. Peterson, A. Tang, \& J.W. Gorter, The Formula for Health and Well-Being in Individuals With Cerebral Palsy: Cross-Sectional Data on Physical Activity, Sleep, and Nutrition, Annals of Rehabilitation Medicine, 44(4) (2020) 301-310. [DOI] [PubMed]

[5] S. Cummings, J. Ayisire, S. Pusch, \& C.G. Pennington, How Can Attention Deficit Hyperactivity Disorder Affect Sport Performance?. Curriculum and Teaching Methodology. 3 (2020) 93-95. [DOI]

[6] C.G. Pennington, \& L.W. Pennington, A Chance to Play is a Chance to Learn: Integrating Individuals with Physical Disabilities into Physical Education, Curriculum and Teaching Methodology, 3 (2020) 81-89. [DOI]

[7] J. McDonald, E. Wind, \& C.G. Pennington, Netball as an Activity for Individuals with Hemophilia, Journal of Physical Education, Recreation \& Dance, 91(2) (2021) 46-48. [DOI]

[8] C. Hernandez, D. Loeung, R. Washington, \& C.G. Pennington, Increasing Physical Activity in Individuals with Spinal Cord Injury, Journal of Physical Fitness, Medicine \& Treatment in Sports, 8(4) (2020) 37-38. [DOI]

[9] O. Verschuren, L. Wiart, D. Hermans, \& M. Ketelaar, Identification of facilitators and barriers to physical activity in children and adolescents with cerebral palsy, The Journal of Pediatrics, 161(3) (2012) 488-494. [DOI] [PubMed]

[10] M.A. Fragala-Pinkham, \& J. Miros, Community Resources: Sports and Active Recreation for Individuals with Cerebral Palsy. In: Miller F., Bachrach S., Lennon N., O'Neil M.E. (eds) Cerebral Palsy. Springer, Cham. (2020) 25072518. [DOI]

[11] J.B. Ryan, A. Katsiyannis, D. Cadorette, J. Hodge, \& M. Markham, Establishing adaptive sports programs for youth with moderate to severe disabilities, Preventing School Failure: Alternative Education for Children and Youth, 58(1) (2014) 32-41. [DOI]

[12] V. Allan, B. Smith, J. Côté, K.A.M. Ginis, \& A.E. Latimer-Cheung, Narratives of participation among individuals with physical disabilities: A life-course analysis of athletes' experiences and development in parasport, Psychology of Sport and Exercise, 37 (2018) 170-178. [DOI]

[13] R.A. Flindall, A Paralympic legacy? British newspaper representations of the Paralympic movement during the 2018 PyeongChang Winter Paralympic Games, Diagoras: International Academic Journal on Olympic Studies, 2 (2018) 145-172.

[14] T.J. Suchomel, S. Nimphius, C.R. Bellon, \& M.H. Stone, The importance of muscular strength: training considerations, Sports Medicine, 48(4) (2018) 765-785. [DOI] [PubMed]

[15] J.M. Ryan, O. Hensey, B. McLoughlin, A. Lyons, \& J. Gormley, Associations of sedentary behaviour, physical activity, blood pressure and anthropometric measures with cardiorespiratory fitness in children with cerebral palsy, PloS One, 10(4) (2015) e0123267. [DOI] [PubMed]

[16] A. Yilmaz, M. Beyleroglu, M. Hazar, H. Kirimoglu, \& U. Sentürk, Flexibility and game actions' effects on lower extremity joint range of motion in cerebral palsied children (spastic type), Studies on Ethno-Medicine, 11(2) (2017) 174-179. [DOI]

[17] J. Toldi, J. Escobar, \& A. Brown, Cerebral Palsy: Sport and Exercise Considerations, Current Sports Medicine Reports, 20(1) (2021) 19-25. [DOI] [PubMed]

[18] T.L. Baldi, G. Spagnoletti, M. Dragusanu, \& D. Prattichizzo, Design of a wearable interface for lightweight robotic arm for people with mobility impairments, International Conference on Rehabilitation Robotics (ICORR) pp. 1567-1573) (2017). [DOI] [PubMed]

[19] D. Keppeler, M. Schwaerzle, T. Harczos, L. Jablonski, A. Dieter, B. Wolf, S. Ayub, C. Vogl, C. Wrobel, G. Hoch, K. Abdellatif, M. Jeschke, V. Rankovic, O. Paul, P. Ruther, T. Moser, Multichannel optogenetic stimulation of the auditory pathway using microfabricated LED cochlear implants in rodents, Science Translational Medicine, 12(553) (2020) eabb8086. [DOI] [PubMed] 


\section{Funding}

No funding was received to carry out this study

\section{Authors Contribution}

Each author contributed equally in the collection of literature, analyses of literature, and the preparation of this article.

Does this article screened for similarity?

Yes.

\section{Conflict of interest}

The authors have no conflicts of interest to declare that they are relevant to the content of this article.

\section{About The License}

(C) The author(s) 2021. The text of this article is open access and licensed under a Creative

Commons Attribution 4.0 International License 